\title{
AN ESTIMATE FOR THE BLOW-UP TIME IN TERMS OF THE INITIAL DATA
}

\author{
JULIO D. ROSSI
}

\begin{abstract}
We find an estimate for the blow-up time in terms of the initial data for solutions of the equation $u_{t}=\left(u^{m}\right)_{x x}+u^{m}$ in $\mathbb{R} \times(0, T)$ and for solutions of the problem $u_{t}=\left(u^{m}\right)_{x x}$ in $(0, \infty) \times(0, T)$ with $-\left(u^{m}\right)_{x}(0, t)=u^{m}(0, t)$ on $(0, T)$ with $m>1$.
\end{abstract}

\section{To Djairo, "El Maestro"}

\section{Introduction.}

In this short note we find an estimate for the blow-up time in terms of the initial data for solutions of the following problems

$$
\begin{cases}u_{t}=\left(u^{m}\right)_{x x}+u^{m}, & (x, t) \in \mathbb{R} \times(0, T), \\ u(x, 0)=u_{0}(x), & x \in \mathbb{R},\end{cases}
$$

and

$$
\begin{cases}u_{t}=\left(u^{m}\right)_{x x}, & (x, t) \in(0,+\infty) \times(0, T), \\ -\left(u^{m}\right)_{x}(0, t)=u^{m}(0, t), & t \in(0, T), \\ u(x, 0)=u_{0}(x), & x \in(0,+\infty) .\end{cases}
$$

For both problems we assume that $m>1$ and $u_{0}$ is nonnegative compactly supported and smooth in its positivity domain.

A remarkable and well known fact is that the solution of parabolic problems can become unbounded in finite time (a phenomena that is known as blow-up), no matter how smooth the initial data are. The study of blow-up solutions has attracted a considerable attention in recent years, see [10], [14] and the references therein. For our problems it is known that all nontrivial solutions blow up in finite time (see [8], [14] for (1.1) and [6] for (1.2)), in the sense that the solution is defined on a maximal time interval, $[0, T)$ with $T<+\infty$ and $\lim _{t \nearrow T}\|u(\cdot, t)\|_{L^{\infty}}=+\infty$.

Key words and phrases. Parabolic equations, blow-up time.

2000 Mathematics Subject Classification. 35K55, 35B40.

Supported by ANPCyT PICT No. 03-00000-00137, CONICET and Fundación Antorchas (Argentina). 
It is interesting to investigate the dependence of the blow-up time with respect to the initial data. For continuity results for the blow-up time as a function of the initial data we refer to [1], [2], [7], [9], [11], [12] and [13].

Our concern here is to obtain bounds for $T=T\left(u_{0}\right)$ in terms of $u_{0}$.

Let us look first to (1.1). The main tool involved in our analysis relies on the natural scaling invariance of the problem. There exits a family (parametrized by $\hat{T}$ ) of self-similar, compactly supported, solutions of the form $u_{\hat{T}}(x, t)=(\hat{T}-t)^{-1 /(m-1)} \varphi(x)$. These solutions $u_{\hat{T}}$ blow up at time $\hat{T}$ and has initial data $u_{\hat{T}}(x, 0)=\hat{T}^{-1 /(m-1)} \varphi(x)$.

Theorem 1.1. The blow-up time $T$ of a solution of (1.1) with initial datum $u_{0}$ verifies

$$
\min _{x}\left(\frac{\varphi}{u_{0}}\right)^{m-1} \leq T \leq \max _{x}\left(\frac{\varphi}{u_{0}}\right)^{m-1} .
$$

The self-similar profile $\varphi(x)$ is a solution of $0=\left(\varphi^{m}\right)^{\prime \prime}(x)+\varphi^{m}(x)-$ $\frac{1}{m-1} \varphi(x)$ that is composed by a finite number of disjoint copies of a radial bump, see [3], [4]. The radial bump is explicit, it takes the form

$$
\varphi(x)=\left(c_{1} \cos ^{2}\left(c_{2} x\right)\right)_{+}^{a},
$$

for some explicit constants $a, c_{1}, c_{2}$, see [14]. Therefore the bounds provided by Theorem 1.1 are computable.

Remark that when the support of $u_{0}$ and the support of $\varphi$ do not coincide then one (or both) of the estimates is immediate.

With the same approach we can prove a similar result for solutions of (1.2). In this case there exists a unique self-similar solution of the form $u_{\hat{T}}(x, t)=(\hat{T}-t)^{-1 /(m-1)} \psi(x)$.

Theorem 1.2. The blow-up time $T$ of a solution of (1.2) with initial datum $u_{0}$ verifies

$$
\min _{x}\left(\frac{\psi}{u_{0}}\right)^{m-1} \leq T \leq \max _{x}\left(\frac{\psi}{u_{0}}\right)^{m-1} .
$$

The profile $\psi$ is explicit and has the form

$$
\psi(x)=c_{1}\left(\left(c_{2}-x\right)_{+}\right)^{a},
$$

see $[5],[6]$.

Finally, we remark that the same approach can be also used to deal with equations involving other operators and/or source terms like $u_{t}=$ $\operatorname{div}\left(|\nabla u|^{q-2} \nabla u\right)+u^{q-1}$ or $u_{t}=u\left(u_{x x}+u\right)$. We only need the existence of a self-similar solution (that comes usually from a scaling invariance law) together with a comparison result. 


\section{Proof of the results.}

Proof of Theorem 1.1. To prove Theorem 1.1 we will make use of the comparison principle that holds for solutions of (1.1).

Let us begin by the lower estimate. Consider the set

$$
A=\left\{\hat{T}: u_{\hat{T}}(x, t) \geq u(x, t) \text { for all } 0 \leq t<\hat{T}\right\} .
$$

By the use of the comparison principle we have that this definition is equivalent to the following

$$
A=\left\{\hat{T}: \hat{T}^{-1 /(m-1)} \varphi(x)=u_{\hat{T}}(x, 0) \geq u_{0}(x)\right\} .
$$

Remark that $A$ is closed. Assume that

$$
\min _{x} \frac{\varphi}{u_{0}}
$$

is positive (otherwise the estimate holds trivially) and let

$$
\underline{T}=\sup A \text {. }
$$

For every $\hat{T}>\underline{T}$ we have that $\hat{T} \notin A$ and then there exists a point $x_{0}$ such that

$$
\hat{T}^{-1 /(m-1)} \varphi\left(x_{0}\right)<u_{0}\left(x_{0}\right) .
$$

Then, every $\hat{T}>\underline{T}$ satisfies

$$
\hat{T}>\left(\frac{\varphi\left(x_{0}\right)}{u_{0}\left(x_{0}\right)}\right)^{m-1} \geq \min _{x}\left(\frac{\varphi}{u_{0}}\right)^{m-1} .
$$

Therefore, we obtain

$$
\underline{T} \geq \min _{x}\left(\frac{\varphi}{u_{0}}\right)^{m-1}
$$

Now we just have to observe that by the definition of $A$ we have $u_{\underline{T}}(x, t) \geq u(x, t)$ for every $0 \leq t<\underline{T}$. Therefore $u(x, t)$ is bounded for $0 \leq t<\underline{T}$ and hence

$$
T \geq \underline{T} \geq \min _{x}\left(\frac{\varphi}{u_{0}}\right)^{m-1} .
$$

This proves the lower bound in (1.3).

To prove the upper bound on $T$ we proceed as before but in this case we have to consider the set

$$
B=\left\{\hat{T}: u_{\hat{T}}(x, t) \leq u(x, t) \text { for all } t \leq T\right\},
$$

which is equivalent to

$$
B=\left\{\hat{T}: \hat{T}^{-1 /(m-1)} \varphi(x)=u_{\hat{T}}(x, 0) \leq u_{0}(x)\right\} .
$$


Let

$$
\bar{T}=\inf B .
$$

As before for any $\hat{T}<\bar{T}$ there must be a point $x_{1}$ with

$$
\hat{T}^{-1 /(m-1)} \varphi\left(x_{1}\right)>u_{0}\left(x_{1}\right) \text {. }
$$

That is

$$
\hat{T}<\left(\frac{\varphi\left(x_{1}\right)}{u_{0}\left(x_{1}\right)}\right)^{m-1} \leq \max _{x}\left(\frac{\varphi}{u_{0}}\right)^{m-1} .
$$

Arguing as before, we get

$$
\bar{T} \leq \max _{x}\left(\frac{\varphi}{u_{0}}\right)^{m-1} .
$$

By the definition of $B$ we conclude

$$
T \leq \bar{T} \leq \max _{x}\left(\frac{\varphi}{u_{0}}\right)^{m-1} .
$$

This shows the upper bound in (1.3) and finishes the proof.

Proof of Theorem 1.2. The proof of Theorem 1.2 is completely analogous to the previous one.

\section{REFERENCES}

[1] P. Baras and L. Cohen. Complete blow-up after $T_{\max }$ for the solution of a semilinear heat equation. J. Funct. Anal. 71, (1987), 142-174.

[2] M. Chaves - J. D. Rossi. Regularity results for the blow-up time as a function of the initial data. Differential Integral Equations. Vol. 17 (11\&12), (2004), 1263-1271.

[3] C. Cortázar, M. del Pino and M. Elgueta. On the blow-up set for $u_{t}=\Delta u^{m}+$ $u^{m}, m>1$. Indiana Univ. Math. J. Vol. 47 (2), (1998), 541-561.

[4] C. Cortázar, M. del Pino and M. Elgueta. Uniqueness and stability of regional blow-up in a porous-medium equation. Ann. Inst. H. Poincaré, Anal. Non Linéaire. Vol. 19 (6), (2002), 927-960.

[5] C. Cortázar, M. Elgueta and O. Venegas. On the Blow-up set for $u_{t}=$ $\left(u^{m}\right)_{x x}, \quad m>1$, with nonlinear boundary conditions. Monatshefte Mathematik, Vol. 142/12, (2004), 67-77.

[6] J. Davila and J. D. Rossi. Self-similar solutions of the porous medium equation in a half-space with a nonlinear boundary condition. Existence and symmetry. J. Math. Anal. Appl. Vol. 296, (2004), 634-649.

[7] C. Fermanian Kammerer, F. Merle, and H. Zaag. Stability of the blow-up profile of non-linear heat equations from the dynamical system point of view. Math. Ann., Vol. 317, (2000), 195-237.

[8] V. A. Galaktionov. Boundary value problems for the nonlinear parabolic equation $u_{t}=\Delta u^{\sigma+1}+u^{\beta}$. Differ. Equations. Vol. 17, (1981), 551-555.

[9] V. A. Galaktionov and J. L. Vazquez. Continuation of blow-up solutions of nonlinear heat equations in several space dimensions. Comm. Pure Appl. Math. Vol. 50, (1997), 1-67. 
[10] V. A. Galaktionov and J. L. Vázquez. The problem of blow-up in nonlinear parabolic equations. Discrete Contin. Dynam. Systems A. Vol 8, (2002), 399433.

[11] P. Groisman, J. D. Rossi and H. Zaag. On the dependence of the blow-up time with respect to the initial data in a semilinear parabolic problem. Comm. Partial Differential Equations. Vol. 28 (3\&4), (2003), 737-744.

[12] M. A. Herrero and J. J. L. Velazquez. Generic behaviour of one-dimensional blow up patterns. Ann. Scuola Norm. Sup. di Pisa, Vol. XIX (3), (1992), 381450.

[13] P. Quittner. Continuity of the blow-up time and a priori bounds for solutions in superlinear parabolic problems. Houston J. Math, Vol. 29 (3), (2003), 757799 .

[14] A. Samarskii, V. A. Galaktionov, S. P. Kurdyumov and A. P. Mikhailov. Blowup in quasilinear parabolic equations. Walter de Gruyter, Berlin, (1995).

Departamento de Matemática, FCeyn., UBA (1428) Buenos Aires, Argentina.

E-mail address: jrossi@dm.uba.ar 